\title{
Legionella makes its mark on histones
}

\section{9 \\ the first \\ demonstration \\ of a bacterial \\ effector \\ that directly \\ modifies host \\ chromatin}

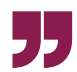

Post-translational modification of histones has a key role in the epigenetic regulation of eukaryotic gene expression, which can be manipulated by pathogens to promote their own survival. Rolando et al. now report that a newly identified Legionella pneumophila methyltransferase generates a unique histone modification that represses host gene expression and enhances intracellular replication of the bacterium.

Owing to its co-evolution with eukaryotic cells, L. pneumophila has a remarkable ability to exploit a range of processes in both its human and protozoan hosts. This is largely achieved by the secretion of effector proteins by the Dot/Icm type IV secretion system (T4SS), and many of these secreted effectors contain eukaryotic-like domains. The authors discovered that the T4SS substrate

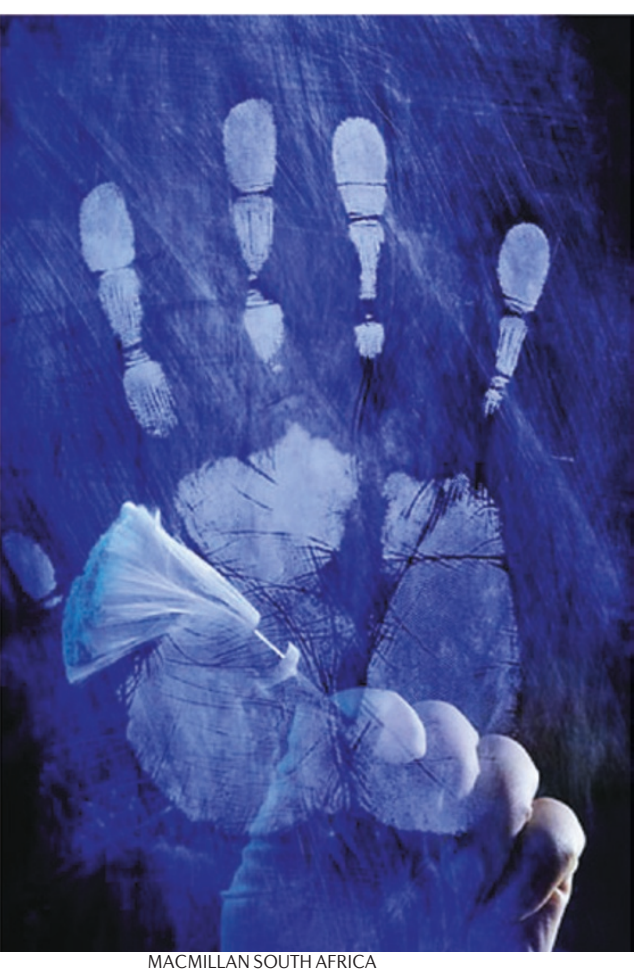
Lpp1683 has a eukaryotic SET domain; these domains are known to catalyse lysine methylation, a histone modification that results in changes in chromatin structure and gene expression. Thus, the authors set out to assess the potential role of Lpp1683 in chromatin remodelling and pathogenesis. Using in vitro histone methyltransferase assays, the authors established that both recombinant and endogenous Lpp1683 catalyses histone $\mathrm{H} 3$ methylation, and the protein was subsequently re-named RomA (regulator of methylation A). Mass spectrometry revealed that lysine 14 of $\mathrm{H} 3$ (H3K14) is the only target of RomA and that the enzyme trimethylates this residue. RomA was also found to be functional in vivo: infection of both human macrophages (THP-1 cells) and protozoan cells with either a wildtype or a RomA-deficient $(\Delta l p p 1683)$ L. pneumophila strain showed that only the wild-type strain induces strong methylation of H3K14. The authors also found that RomA contains a nuclear localization signal and localizes predominantly to the nucleus, where it is enriched at heterochromatic regions (which typically contain genes that are transcriptionally inactive).

H3K14 is usually acetylated, and together with H3S10 phosphorylation and $\mathrm{H} 3 \mathrm{~K} 9$ acetylation, this modification is associated with transcriptional activation. Because methylation of H3K14 could potentially inhibit its acetylation, the authors hypothesized that RomA exerts transcriptional repression. To investigate this, a model cell line was constructed that contained artificial RomA-tethering sites upstream of the luciferase reporter gene. Transfection with wild-type RomA or a catalytically inactive mutant allowed an assessment of the effect of RomA on both transcription (by measuring luciferase activity) and chromatin modifications (by using chromatin immunoprecipitation (ChIP)).

Wild-type RomA strongly repressed luciferase activity, whereas the inactive variant did not, indicating that transcriptional inhibition is mediated by the methyltransferase activity of RomA. ChIP experiments also showed that recruitment of RomA to the luciferase promoter leads to an increase in H3K14 methylation and a consequent decrease in H3K14 acetylation. Moreover, ChIP-sequencing analyses of L. pneumophila-infected THP-1 cells identified 4,870 promoters that were enriched for methylated H3K14, suggesting that RomA activity occurs on a genome-wide scale. Interestingly, H3K14 methylation was particularly pronounced at the promoters of immune genes, such as those encoding interleukin- 6 and tumour necrosis factor, and the authors confirmed that RomA represses the transcription of these genes.

Finally, the authors showed that the intracellular replication rate of the $\Delta l p p 1683$ strain was substantially slower than that of wild-type L. pneumophila. However, plasmid-borne lpp1683 rescued the growth defect, confirming that RomA promotes intracellular replication. Together with the observed inhibitory effects on immune gene expression, these findings suggest that RomA promotes pathogenesis by enhancing bacterial growth and manipulating the host immune response.

Although chromatin remodelling by bacterial pathogens has been observed previously, this is the first demonstration of a bacterial effector that directly modifies host chromatin. Furthermore, because this is a unique modification, it seems that L. pneumophila has evolved a very specific strategy to promote its own replication in the host.

Christina Tobin Kåhrström

ORIGINAL RESEARCH PAPER Rolando, M. et al. Legionella pneumophila effector RomA uniquely modifies host chromatin to repress gene expression and promote intracellular bacterial replication. Cell Host Microbe 13, 395-405 (2013) 